therapies have been reported e.g. tofacitinib [3] and plasma exchange therapy (PE) $[1,4,5]$.

Objectives: We evaluated the effect of plasma exchange (PE) on survival in patients with refractory RP-ILD who were positive for anti-MDA5 antibodies. Methods: Among 167 patients newly diagnosed with PM/DM, clinically amyopathic DM, or cancer associated myositis from 2008 to 2019 at our hospital, 12 were diagnosed with refractory RP-ILD and were positive for anti-MDA5 antibodies. PE was used as an adjunct to standard therapy and consisted of fresh frozen plasma as replacement solution. The primary outcome was non-disease-specific mortality. anti-MDA5 antibody titres were measured by ELISA using the MESACUP anti-MDA5 test in 155 patients whose serum was frozen and stored at the time of diagnosis.

Results: Anti-MDA5 antibodies were detected in 35 patients, of whom 26 were diagnosed with RP-ILD and 11 were refractory to intensive immunosuppressive therapy. Seven patients received PE (PE group) and four did not (non-PE group). The 1-year survival rate of the PE group was higher than that of the non-PE group $(100 \%$ and $25 \%$, respectively, $P=0.011)$. Regarding adverse events associated with PE, two patients had anaphylactic shock, one had high fever due to fresh frozen plasma allergy and one had a catheter infection. All adverse events resolved with appropriate treatment.

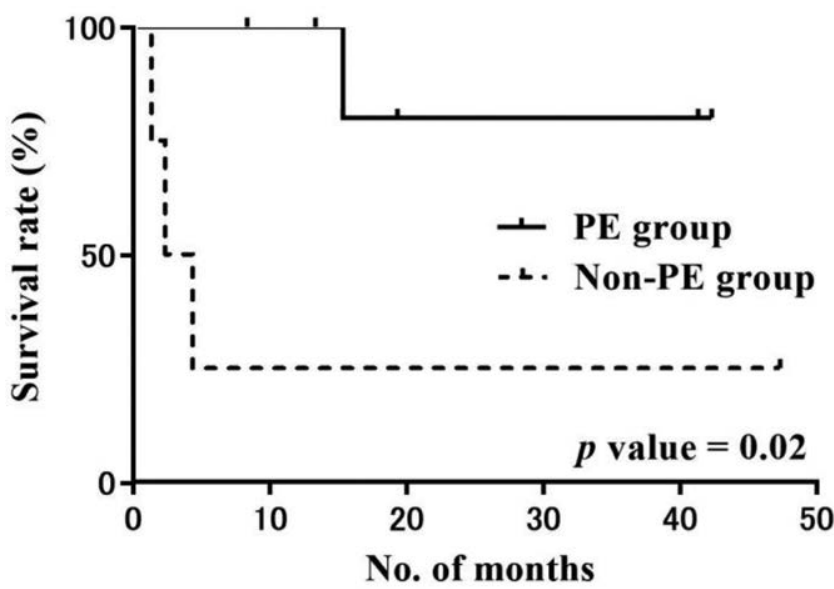

Conclusion: We evaluated the association between 1-year survival rate and PE for refractory RP-ILD in patients positive for anti-MDA5 antibodies. Intensive immunosuppressive therapy improved the survival rate in RP-ILD patients with anti-MDA5 antibodies, but $20-30 \%$ of cases were still fatal. PE could be administered to patients with active infectious disease who were immunocompromised by intensive immunosuppressive therapy. PE may be considered in refractory RP-ILD patients positive for anti-MDA5 antibodies.

References:

[1] Nakashima R, Hosono Y, Mimori T. Clinical significance and new detection system of autoantibodies in myositis with interstitial lung disease. Lupus. 2016;25:925-33.

[2] Kurasawa K, Arai S, Namiki Y et al. Tofacitinib for refractory interstitial lung diseases in anti-melanoma differentiation-associated 5 gene antibody-positive dermatomyositis. Rheumatology. 2018;57:2114-9.

[3] Silveira MG, Selva-O'Callaghan A, Ramos-Terrades $\mathrm{N}$ et al. Anti-MDA5 dermatomyositis and progressive interstitial pneumonia. QJM. 2016;109:49-50.

[4] EndoY, Koga T, Suzuki T et al. Successful treatment of plasma exchange for rapidly progressive interstitial lung disease with anti-MDA5 antibody-positive dermatomyositis: A case report. Medicine (Baltimore). 2018;97: e0436.

[5] Abe $\mathrm{Y}$, Kusaoi M, Tada $\mathrm{K}$ et al. Successful treatment of anti-MDA5 antibody-positive refractory interstitial lung disease with plasma exchange therapy. Rheumatology. 2019.

Acknowledgments: Funding: This work was supported by Japan Society for the Promotion of Science KAKENHI Grant Number JP18K15433.

Disclosure of Interests: None declared

DOI: 10.1136/annrheumdis-2020-eular.1920

\section{THU0338 \\ THE CIRCULATING CD19-POSITIVE LYMPHOCYTES IN PATIENTS WITH SYSTEMIC SCLEROSIS: MODULATION WITHIN A YEAR AFTER THE INITIATION OF RITUXIMAB THERAPY}

L. P. Ananyeva ${ }^{1}$, L. Garzanova ${ }^{2}$, O. Koneva ${ }^{2}$, M. Starovoytova $^{2}$, O. Desinova ${ }^{2}$,

O. Ovsyannikova ${ }^{2}$, M. Cherkasova ${ }^{3}$, A. Aleksankin ${ }^{3}{ }^{1}$ V. A. Nasonova Research
Institute of Rheumatology, Microcirculation and Inflammation, Moscow, Russian Federation; ${ }^{2}$ V. A. Nasonova Research Institute of Rheumatology, Moscow, Russian Federation; ${ }^{3}$ V. A. Nasonova Research Institute of Rheumatology, Immunology, Moscow, Russian Federation

Background: Significant disorders of B-cell homeostasis have been detected in systemic sclerosis (SSc) $[1,2]$. The improvement of the disease with anti-CD20 monoclonal antibody rituximab (RTM) has been shown in SSc [3]. There are limited data on change in circulating B lymphocytes count after RTM treatment in patients with SSc.

Objectives: to investigate the modulations in absolute and relative numbers of circulating CD19-positive B lymphocytes (B-lymph) in patients with SSc within a year after the initiation of RTM therapy.

Methods: 71 pts with SSc were included in the prospective study. Mean age was $46 \pm 13$ yrs., $83 \%$ were women, $59 \%$ had diffuse subset. Duration of SSC from the first non-Raynaud`s symptom was $5.6 \pm 4.4$ yrs. All pts received low doses of glucocorticoids and $45 \%$-immunosuppressive medications. The average follow-up of patients was $13.2 \pm 2.0$ (11-18) months. The mean dose of RTM for the period of follow up was $1.43 \pm 0.60$ grams, 48 patients received $<$ $2 \mathrm{~g}$ of RTM (group 1, mean of $1.1 \pm 0.1 \mathrm{~g}$ ) and 23 patients received $\geq 2$ grams of RTM (group 2, mean dose of $2.2 \pm 0.6 \mathrm{~g}$ ). Peripheral blood CD19-positive cell count was obtained by flow cytometry in patients and in 20 healthy persons, comparable in sex and age. Data are presented as the percentage ( $\mathrm{P} \%)$ and absolute number (AN) of B-lymph per $\mathrm{ml}$ of blood. In patients, the number of B-lymph was determined before ( $n=67$ pts), within first month after the first introduction of RTM $(n=66), 6$ months later $(n=34)$ and at the end of the study $(\mathrm{n}=71)$

Results: At baseline, the AN and P\% of B-lymph in pts did not differ from the healthy control. In pts with short disease duration ( $\leq 3 \mathrm{yrs}$.) the number of B-lymph before treatment with RTM was the higher (compared with longer duration $>3 \mathrm{yrs}$ ) those who was ill $\geq 3$ yrs.) and there was negative correlation between $B$-lymph count and duration of the disease $(R-0.36, p=0.003$ for $A N$ and $R-0.48, p=0.001$ for $P \%)$. The number of $B$-lymph was significantly lower in patients receiving cyclophosphamide (Cyc) before being started with RTM. There was a negative correlation between the AN of B-lymph and the cumulative dose of Cyc $(R-0.293, p=0.016)$. In 1 month after the initiation of RTM a complete depletion of B-lymph was observed in all pts and in six months it persisted in $79 \%$ of cases, the rest began to repopulate $(15 \%)$ or reached a normal levels $(6 \%)$. At the end of the follow up the number of B-lymph was significantly lower than before treatment and a complete $(n=41$ pts) or partial $(n=23)$ depletion of B-lymph remained, and only in $7(10 \%)$ pts the count of this cells was normalized. We revealed a negative correlation between the AN of B-lymph and the cumulative dose of RTM $(R-0.237, p=0.048)$. Higher doses of RTM in group 2 induced a more significant depletion than in group 1. Change in forced vital capacity and diffusing capacity of the lung (\% predicted) during follow up were less pronounced for pts in group 1 compared with group $2(\Delta \mathrm{FVC} 2,4 \%$ and $7,5 \% \mathrm{p}=0,01 ; \Delta \mathrm{DLCO}-0,35 \%$ and $5,05 \%, \mathrm{p}=0,001$, respectively).

Conclusion: RTM may be more effective at the early stage of the disease, when the level of B-lymph is the highest. In SSc, the repopulation of B-lymph after depletion with RTM develops slowly. There were a more significant depletion of B-lymph and a more pronounced improvement in pulmonary function with the higher dose of RTM to compare with the lower one. This results indicate the option of a flexible dosing regimen of RTM.

References:

[1] Sanges S. et al. La Revue de médecine interne 38 (2017) 113-124

[2] Forestier A. et al. Autoimmunity Reviews 17 (2018) 244-255

[3] Jordan S, et al. Ann Rheum Dis 2015;74:1188-1194. doi:10.1136/ annrheumdis-2013-204522

Disclosure of Interests: None declared

DOI: 10.1136/annrheumdis-2020-eular.1419

\section{THU0339 PROGNOSIS OF DIGITAL ULCERS IN PATIENTS} WITH SSC.

S. Barsotti ${ }^{1}$, V. Venturini ${ }^{1}$, M. DI Battista ${ }^{1}$, S. Macchiarulo ${ }^{1}$, A. Della Rossa ${ }^{1}$, M. Mosca ${ }^{1} .{ }^{1}$ Rheumatology Unit - Pisa University Hospital, Pisa, Italy

Background: Digital ulcers (DUs) are one of the main burdens in patients with systemic sclerosis (SSc) as they have a major impact on quality of life and prognosis. Some DUs are associated with the presence of subcutaneous calcinosis (SC) that may worsen their management, and the prognosis of these DUs is still not well defined.

Objectives: To define the characteristics of SSc patients with DUs related to SC and analyze the impact on prognosis and on healing time. 